\title{
The Application of Artificial Intelligence in the Genetic Study of Alzheimer's Disease
}

\author{
Rohan Mishra', Bin Li ${ }^{1,2 *}$ \\ ${ }^{1}$ Washington Institute for Health Sciences, Arlington, VA 22203, USA \\ ${ }^{2}$ Georgetown University Medical Center, Washington D.C. 20057, USA
}

[Received January 2, 2020; Revised March 8, 2020; Accepted March 12, 2020]

\begin{abstract}
Alzheimer's disease (AD) is a neurodegenerative disease in which genetic factors contribute approximately $70 \%$ of etiological effects. Studies have found many significant genetic and environmental factors, but the pathogenesis of AD is still unclear. With the application of microarray and next-generation sequencing technologies, research using genetic data has shown explosive growth. In addition to conventional statistical methods for the processing of these data, artificial intelligence (AI) technology shows obvious advantages in analyzing such complex projects. This article first briefly reviews the application of AI technology in medicine and the current status of genetic research in AD. Then, a comprehensive review is focused on the application of $\mathrm{AI}$ in the genetic research of $\mathrm{AD}$, including the diagnosis and prognosis of $\mathrm{AD}$ based on genetic data, the analysis of genetic variation, gene expression profile, gene-gene interaction in $\mathrm{AD}$, and genetic analysis of AD based on a knowledge base. Although many studies have yielded some meaningful results, they are still in a preliminary stage. The main shortcomings include the limitations of the databases, failing to take advantage of AI to conduct a systematic biology analysis of multilevel databases, and lack of a theoretical framework for the analysis results. Finally, we outlook the direction of future development. It is crucial to develop high quality, comprehensive, large sample size, data sharing resources; a multi-level system biology AI analysis strategy is one of the development directions, and computational creativity may play a role in theory model building, verification, and designing new intervention protocols for $A D$.
\end{abstract}

Key words: Alzheimer's disease, genetics, artificial intelligence, machine learning

\section{Introduction}

Alzheimer's disease (AD) is a neurodegenerative disease characterized by progressive exacerbation of dementia; finally, patients lose the ability to respond to their environment. Except for cholinesterase inhibitors and memantine, which can alleviate or stabilize symptoms for a limited time, there is currently no way to stop the progression of $\mathrm{AD}$ [1]. With the aging of the world's population, AD not only causes more personal and family suffering, but caregivers of patients with $\mathrm{AD}$ have a higher prevalence of depression and anxiety, and $\mathrm{AD}$ becomes a serious social burden [2]. It is estimated that currently one in 10 Americans (10\%) aged 65 and older has $\mathrm{AD}$. In 2019, there were approximately 5.8 million AD patients in the United States. By 2050, this number may grow to a projected 13.8 million in the US, and the number of people living with dementia will reach 131.5 million worldwide [3-5]. Based on the age of onset, $\mathrm{AD}$ is classified as early-onset $\mathrm{AD}$ (EOAD) and late-onset $\mathrm{AD}$ (LOAD). EOAD accounts for approximately $5 \%$ of all $\mathrm{AD}$ cases and occurs in patients under 65 years old. Among them, less than half of the patients carry a causal mutation that manifests as an autosomal dominant

*Correspondence should be addressed to: Dr. Bin Li, MD, Georgetown University Medical Center, 3900 Reservoir Road, NW, Washington DC, United States. E-mail: bl444@georgetown.edu.

Copyright: ( 2020 Mishra R et al. This is an open-access article distributed under the terms of the Creative Commons Attribution License, which permits unrestricted use, distribution, and reproduction in any medium, provided the original author and source are credited. 
inheritance pattern, known as early-onset familial AD. LOAD accounts for approximately $95 \%$ of all $\mathrm{AD}$ cases and occurs in patients over 65 years old. Based on the existence of family aggregation, $\mathrm{AD}$ can also be divided into familial and sporadic cases. Familial cases are mainly EOAD, but there are also cases of LOAD. More than $90 \%$ of $\mathrm{AD}$ patients are sporadic cases that are usually also LOAD [6]. The etiology for most cases of $\mathrm{AD}$ remains unclear and is thought to be the result of a complex interaction between genetic and environmental factors involved in neurobiological and immunological processes $[7,8]$, but an estimated $70 \%$ of the risk is attributable to genetic factors [9-12]. Currently, the amyloid hypothesis is a prevalent theory of the pathogenesis of AD. This hypothesis holds that a variety of factors cause imbalance in the production and clearance of $\beta$-amyloid, leading to the accumulation of $\beta$-amyloid in the brain, which in turn leads to neuroinflammation and the formation of neurofibrillary tangles in neurons, that will eventually lead to neuronal dysfunction and death, but the detailed pathological process is unclear [13].

One of the important goals of medical research is to discover the genetic and environmental factors that cause disease, namely, etiology studies, and the results can provide clues for researching the prevention and treatment of AD. Since the 17th century, Newton's methodology has been widely used in scientific research, including medical research, which emphasizes that the world's apparent complexity can be solved by analyzing phenomena and simplifying them into the simplest components. In fact, we have achieved great success by utilizing this methodology. In the medical field, we have been able to successfully prevent and treat many diseases caused by one or several factors. For example, smallpox has been completely eliminated on Earth with the use of vaccines. However, currently, there are no solutions for the prevention and reversible treatment of certain complex diseases, such as $\mathrm{AD}$, mainly because these diseases involve complex interactions between many factors, and the complexity of humans makes it impossible to use a simplified model to understand these diseases $[14,15]$. In recent years, the rapid development of artificial intelligence (AI) technology has provided an opportunity to solve these problems involving massive data and ultracomplex structures that exceed the processing capabilities of the human brain [16-18]. In the field of AI, $\mathrm{AD}$ ranked fourth among all diseases in terms of the number of AI studies conducted [19]. AI adopts an integrative approach and model neurobiological components as functional modules of pathophysiology embedded within the complex, social dynamics that influence the phenomenology of neuropsychiatric disorders [20]. Because genetic factors are the main cause of most $\mathrm{AD}$ cases, they have been the focus of $\mathrm{AD}$ pathogenesis research. In recent years, with the widespread use of microarray and next-generation sequencing technologies, research using genetic data has shown explosive growth. This situation urgently requires the help of AI technology. Currently, genetic research on $\mathrm{AD}$ using AI technology is continuously increasing. Therefore, this article has conducted a comprehensive review of the research in this field and provides a perspective on the direction of future developments.

\section{Artificial Intelligence}

The use of tools could be considered to be an "extension" of the human body's natural function. In the same way, computers can act as "extensions" of the human brain's functionality. With the rapid development of computer power, the acquisition of large amounts of data, and the theory of computation, AI has or almost "will surpass human performance in several domains" [21, 22]. Intelligence is and has been humanity's most useful ability for thriving on earth. There is reason to believe that with the development of AI technology, human productivity will enter a new era. AI is used in a broader scope with many definitions from different perspectives, and the ones that are commonly accepted include that AI is a branch of computer science that enables computers to perform tasks that generally require human intelligence; another perspective is that AI is a system that perceives the environment and takes action with a maximal possibility to complete a task [23]. To achieve "intelligent" functions, a large number of algorithms, methods, or strategies have been developed, and the main approaches of AI technology are problem solving by searching, knowledge-based reasoning and planning, uncertain knowledge-based reasoning, and learning from examples. The methods or strategies of problem solving by searching include uninformed or heuristic searches, local searches, optimizations, evolutionary computations, and adversarial searches. Knowledge-based reasoning and planning include logic programming, automated reasoning and ontological engineering. Uncertain knowledge-based reasoning includes Bayesian networks, hidden Markov models, Kalman filters, a utility theory, and decision networks. Learning from examples is based on a mathematical/statistical classification and machine learning. Among them, machine learning is the most widely used AI technique in academia and industry [24, 25].

Machine learning is a subset of AI that focuses on designing a computer program that automatically improves through experience. It works with datasets by examining the data and learning patterns within it. Most machine learning approaches fall into two categories: supervised and unsupervised learning algorithms. 
Supervised learning algorithms use labeled data, i.e., training data that have the correct result given an input, and these are best for classification and regression tasks. The common algorithms include artificial neural networks, Bayesian networks, support vector machines, decision trees, random forests, and K-nearest neighbors. In contrast, unsupervised learning algorithms use unlabeled data, where the algorithm needs to find and learn inherent patterns within the dataset. The common algorithms include K-means, distance clustering, density clustering, hierarchical clustering and Markov chain. There are also some algorithms that combine supervised and unsupervised learning, for example, reinforcement learning [24, 26, 27]. Deep learning is an even more specific subset of AI and machine learning. Deep learning is a machine learning algorithm that simulates a human brain approach to solving problems. It consists of several "layers" each with a various number of nodes all interconnected in a network. Information is input into the first "layer" and goes through several linear transformations until it outputs a result at the end. It can be supervised, unsupervised or enhanced, depending on how it is applied [28]. Before AI, many projects were implemented with complicated rule-based algorithms, which would continuously grow more complicated as more anomalies were discovered in the data. We can continuously make more rules and algorithms to try and account for every possible oddity, but this becomes laborious and overcomplicated. However, a machine learning implementation can simply learn these patterns. Furthermore, machine learning will be able to discover additional patterns of increased complexity or abstraction within the data. As the quantity, quality, and diversification of data increases, the effectiveness of a machine to learn patterns and logic in it becomes more efficient. This expansion of data is especially noticeable in medicine because of the new approaches to collect large amounts of biological data, such as genomic and other omics biology datasets [29]. Therefore, AI will have a great future in the application of healthcare, including the prevention, detection, diagnosis, and treatment of diseases, the management of health systems, and the development of medical research $[30,31]$.

\section{The Applications of AI in Medicine}

Shortly after the official naming of AI at a Dartmouth College meeting in 1956, research into the application of $\mathrm{AI}$ in the medical field began. The main research topic at that time was the Medical Diagnostic Decision Support System (MDDS). For example, Warner et al. developed a pioneering MDDS system that can be used to diagnose congenital heart disease $[32,33]$. There was a period of low amounts of AI research from 1974 to 1993; however, due to the remarkable progress of computers and information technology in recent years, research on the application of $\mathrm{AI}$ in the medical field has become a hot topic [24]. Many information technology companies and research institutions are currently working on the development of AI technology in clinical medicine, health systems management, public health, and medical research. For clinical medicine, the development of computer vision, computer analysis of images and videos, and the combination of these with AI technology have significantly improved the classification and detection of images, and this is very beneficial for medical imaging. These technologies have been developed for imaging diagnosis in radiology [34], pathology [35], dermatology [36], ophthalmology [37], cardiology [38], neurology [39], gastroenterology [40], and surgery [41] have shown satisfactory results in many aspects. In addition, AI can predict the progress of disease and treatment effects by learning the health trajectory from a large number of patients. For instance, by using ${ }^{18} \mathrm{~F}$-fluorodeoxyglucose PET of the brain, a deep learning algorithm for the early prediction of $\mathrm{AD}$ was developed, and this achieved $82 \%$ specificity and $100 \%$ sensitivity at an average of 75.8 months prior to the final diagnosis [42]. Therefore, it is believed that the use of AI technology in clinical facilities may help improve the quality of medical practice, which is particularly helpful for physicians with insufficient training or experience, especially in developing countries with insufficient medical resources [43]. AI technology can also expand the reach of medical services; for example, patients can use a smartphone or smart watch application for self-care, and some applications are currently approved by the FDA. Precision medicine is based on the patient's genetics, environment, and lifestyle factors and is tailored to his or her personal healthcare plan and the clinical decisions for the patient. AI technology can analyze and process very large amounts of genetics, environment and lifestyle data, and this allows for the ability of precision medicine to be applied in clinical practice. In addition, it may play an important role in health system management and public health $[17,27$, 30, 44-46].

In the field of biomedical research, currently developed biological and medical technologies can extract a large amount of laboratory and clinical research data from complex biological processes, such as genomes, transcriptomes, proteomics, cytological images, chemical and biological macromolecular structures, interaction information, and clinical data from electronic medical records. AI technology can analyze and process large and complex biological data to help clarify the corresponding physiological and pathological mechanisms and then assist in designing and screening drug molecules and in designing and analyzing clinical trials. For research at the 
gene level, AI can help to predict the binding affinities of transcription factors, DNA- and RNA-binding proteins, cis-regulatory/enhancer elements, DNA methylation sites, histone modifications, chromatin accessibility, transcription start sites, tissue-regulated splicing, special gene expression and translation efficacies, transcriptome patterns in a particular cell or condition, microRNA precursors and binding targets, variant calling, functional consequences of noncoding variants, and coding variants pathogenicity. AI can also help to identify long noncoding RNAs, generate protein-coding DNA sequences, and design DNA probes for protein binding microarrays. As the amount of genomic data grows exponentially, deep learning seems to be the best way to analyze these data sources and complete genomic modeling tasks; however, the prediction of complex human disease phenotypes is currently far from mature [47-50]. For protein level research, current AI technologies can predict the secondary structure, solvent-accessible surface area, solvent accessible surface area, protein contact maps, and disordered regions; however, the prediction of the tertiary protein structure is still challenging [51, 52]. For cell- and tissue-level research, automated high-content, highthroughput imaging technology is not only a powerful tool for studying biomedical issues but also a tool used for all stages for developing target-based drugs. Specific functions of AI in image processing include signal denoising and enhancement, segmentation, label-less imaging, live cell imaging, imaging-based phenotype, single cell tracking, and modeling of reconstructed pedigree trees $[53,54]$. With the application of AI technology in the chip laboratory, cell-based or organoidbased assays, and automatic compound synthesis, it is possible to greatly accelerate the development of new drugs. AI can be used to analyze literature and highthroughput compound screening data and to propose plans for initial molecular screening and automated chemical synthesis. After obtaining bioassay data, by updating the machine learning model, a new molecular optimization plan can be proposed, and the bioassay can be performed again. In this way, an automated drug development cycle based on AI design and high-throughput bioassay is formed [55]. Drug repurposing is a low-cost, rapid drug development pathway. AI technology can predict drug repurposing by analyzing large-scale transcriptomics, molecular structure data, and clinical databases [56]. Clinical trials are the bottleneck of new drug development, and researchers believe that the use of AI technology in the design and implementation of clinical trials can help solve this problem. By analyzing patient genetic and clinical data, AI can help select a subset of the population who may be sensitive to new drugs, and it can also help recruit subjects by matching patients and clinical trials. Combining AI technology with wearable sensors and noninvasive diagnostics during clinical trials can provide a mobile, real-time patient monitoring system and may predict the dropout risk of a particular patient [57]. Although research on AI-based medical technology has developed rapidly and has broad application prospects, there are few examples of clinical applications. It can be said that AI-based medical technology is still in its infancy $[30,58]$.

\section{Overview of Genetics Research on AD}

It is estimated that for $\mathrm{AD}$ cases other than early-onset familial $\mathrm{AD}$, genetic factors may contribute at approximately $70 \%$ of the etiologic role $[9,10]$. The genetic variations between individuals in the population include single nucleotide variations (SNVs), tandem repeat variations, small insertions and deletions, large segment deletions and duplications (copy number variations), chromosome rearrangements (duplication, deletion, inversion, and translocation), and aneuploidy or polyploidy (often causing major genetic diseases) [59]. The human genome is approximately $3.2 \times 10^{9}$ base pair (bp), of which the noncoding region accounts for approximately $99 \%$ of the entire human genome. The noncoding region also has important cellular regulatory functions and includes regulatory elements (promoters, enhancers, silencers, and insulators); production of transfer RNA, ribosomal RNA, microRNAs; long noncoding RNAs; formation of structural elements of the chromosome, including telomeres, satellite DNA, etc. $[50,60,61]$. To discover genetic variations associated with the development of $\mathrm{AD}$ in the human genome, four strategies have been applied: genetic linkage analyses, candidate gene/pathway association studies, genomewide association studies (GWAS), and next-generation sequencing (NGS)-based association studies [62].

Genetic linkage analysis is one of the earliest strategies for assessing the genetic basis of Mendelian traits. It maps genetic loci through genetic markers and segregation analysis in pedigrees [63]. Through genetic linkage analysis, causal mutations in three genes, amyloid precursor protein $(A P P)$, presenilin 1 (PSEN1), and presenilin 2 (PSEN2) were found in early-onset familial AD [57]. They are located on chromosomes 21, 14, and 1, respectively [64]. Individuals with Down syndrome carry an extra copy of chromosome 21 , which is responsible for the pathological changes of EOAD [65].

Candidate gene/pathway approaches are small-scale and low-resolution association studies based on existing knowledge of some genes. This approach has identified the apolipoprotein $\mathrm{E}$ gene (APOE) alleles as risk factors for late-onset AD. Although this method is not commonly used today, it is still valuable depending on the gene or 
population, for example, when exploring polymorphisms with low allele frequencies [66, 67].

Based on advances in microarray technology, GWAS can assess the association of thousands of single nucleotide polymorphisms (SNPs) of a disease and provide information on genetic variations associated with the risk of certain diseases [68]. Certain international cooperation projects, such as the International Alzheimer's Disease Project (IGAP), have conducted large GWAS samples of LOADs involving tens of thousands of patients $[69,70]$.

GWAS has confirmed that $A P O E \& 4$ is the most important genetic risk factor for $\mathrm{AD}$, but more susceptible loci have been reported recently and include $A B C A 7$, ACE, ADAM10, ADAMTS1, ATP5H-KCTD2, BIN1, BZRAP1, CASS4, CD2AP, CD33, CELF1, CLU, COBL, CR1, DSG2, EPHA1, FBXL7, FERMT2, FRMD4A, HBEG F, HLA-DRB5-HLA-DRB1, INPP5D, IQCK, MEF2C, MS4A4E/MS4A6A, MTHFDIL, NME8, PFDN1HBEGF, PICALM, PTK2B, RANBP2, SCIMP, SLC1OA2, SLC24A4-RIN3, SORL1, SPPL2A, TREM2, TRIP4, USP6NL-ECHDC3, WWOX and ZCWPWI [11, 62, 71]. The GWAS data are available from the National Human Genome Research Institute - European Bioinformatics Institute (NHGRI-EBI) GWAS catalogue (www.ebi.ac.uk (gwas/).

NGS uses revolutionary massively parallel sequencing technology that allows each base in the entire human genome to be quickly sequenced multiple times to provide comprehensive and accurate DNA data. Sequencing of all protein coding regions is called wholeexome sequencing (WES), and sequencing of the entire genome is called whole-genome sequencing (WGS) [72]. Since GWAS requires a large number of samples to achieve statistical significance, it is difficult to find extremely rare variants that affect the risk of $\mathrm{AD}$, but NGS can obtain subjects' complete genomic sequence data; therefore, it is possible to capture all such variants. NGS has led to the discovery of a few mutant genes in some cases with unexplained EOAD, and includes NOTCH3, SORL1, TREM2, etc. [73-75]. In some very small population groups, NGS has also discovered a number of susceptibility loci related to the development of $\mathrm{AD}$, but GWAS usually missed them, such as $A R S A, C H M P 2 B$, CSF1R, FSIP2, GRN, IGHG3, NCSTN, NOS1AP, PLD3, TM2D3, TTC3, ZBTB4, and ZNF655 [76-82].

With the exception of $A P O E$, all of the genetic variations described above have little effect on the pathogenesis of $\mathrm{AD}$. To understand the etiology of $\mathrm{AD}$ other than early-onset familial AD, it might be necessary to consider the effect of multiple variants (additive effects), epistasis (multiplicative effects), and the interaction of genes with the environment. Genetic risk scores can be used to describe the synthetic effects of multiple variants on the pathogenesis of $\mathrm{AD}$ by calculating the number of disease-related alleles and their power to predict the risk of AD. A genetic risk score study based on an SNP dataset including 3,049 AD cases and 1,554 controls showed that the maximum predictive accuracy for $\mathrm{AD}$ was $82 \%$ [83]. In epistasis studies, interactions were found in some genes that have never been associated with $\mathrm{AD}$ before, while the individual gene analyses did not show any effect, for example, the interacting SNP pair in KHDRBS2 and CRYL1 [84]. The results support that the epistasis effect also contributes to a portion of the heredity of AD. A review article by Raghavan and Tosto summarizes studies of gene-to-gene interactions in $\mathrm{AD}$ [85]. The concept of functional genomics attempts to link omics data such as genomics, transcriptomics, proteomics, and metabolomics to explain the complex process from genotypes to phenotypes at a comprehensive level. Functional pathway analysis showed that AD-related genetic variants were mainly enriched in APP metabolism, inflammation, lipid metabolism, tau protein binding, endocytic/vesicularmediated transport and synaptic function pathways [11, $62,70,71,86]$.

Many environmental factors have been found to increase the risk of $\mathrm{AD}$, including brain trauma, low education levels, risk factors for cardiovascular disease, lifestyle (e.g., tobacco, alcohol consumption, exposure to greenery, and exercise) [87], air pollution [88], heavy metal exposure (e.g., mercury, manganese) [89, 90], pesticide exposure, etc. However, there is no evidence showing that these environmental risk factors alone are the cause of $\mathrm{AD}$; it is speculated that they may trigger the pathogenesis of $\mathrm{AD}$ by interacting with the risk genes of an individual. Studies have evaluated the role of $A P O E$ genes in interacting with the environment. For example, if individuals have both the $A P O E \& 4$ allele and low physical activity, their risk of developing dementia is much higher than those having only one factor [91]. However, there is very little research data on the interaction between genetic variations and environmental risk factors [92].

Epigenetics focus on mechanisms that affect gene expression without altering DNA sequences, including DNA methylation, histone modifications, chromatin remodeling and noncoding RNA regulation. Epigenetic modifications are influenced by external and internal environmental factors, such as age, lifestyle, disease status, pollution [93]. Many studies have reported epigenetic changes in the brains of $\mathrm{AD}$ patients, for example, changes in DNA methylation levels in COASY, SPINT, BRCA1, and PLD3 promoter regions, APOE CpG islands and HOXA gene clusters, an overall reduction in DNA methylation in the cortex and hippocampus, increased histone deacetylase (HDAC) 6, decreased class III HDACs, changes in miRNAs (miR-29 family, 
miRNA-7, miRNA-9-1, miRNA-23a/miRNA-27a, miRNA-34a, miRNA-125b-1, miRNA- 146a, and miRNA-155) and long noncoding RNA (BC200 RNA) levels in the AD brain. These findings have been summarized in some reviews [62, 94].

It has been reported that some mitochondrial haplogroups and single nucleotide polymorphisms affect the risk of $\mathrm{AD}$, but due to the small sample size and lack of validation, the contribution of mitochondrial genetic variation to AD risk is inconclusive [95, 96].

As mentioned above, most cases of AD are thought to be caused by complex interactions between multiple genetic variations and environmental risk factors. Highquality large-scale databases and powerful bioinformatics methods may be the main ways to help understand this complex interaction, specifically the global-scale AD research shared database and AI technology. Some important data sharing resources in $\mathrm{AD}$ research are: Alzheimer's Disease Genetics Consortium (ADGC, www.adgenetics.org), Alzheimer's Disease Sequencing Project (ADSP, www.niagads.org/adsp/content/home), Alzheimer's Disease Neuroimaging Initiative (ADNI, http://adni.loni.usc.edu/), AlzGene (www.alzgene.org), Dementias Platform UK (DPUK, https://portal. dementiasplatform.uk/), Genetics of Alzheimer's Disease Data Storage Site (NIAGADS, www.niagads.org/), Global Alzheimer's Association Interactive Network (GAAIN, www.gaain.org/), and National Centralized Repository for Alzheimer's Disease and Related Dementias (NCRAD, https://ncrad.iu.edu) [97, 98].

\section{The Applications of AI in AD Genetic Analysis}

AI technology, mainly machine learning algorithms, has shown to be efficient for large data analysis of highdimensional complex systems. Currently, machine learning has been used in the studies of diagnosis and prognosis of AD based on genetic data, the analysis of genetic variations, gene expression profiles, gene-gene interactions in $\mathrm{AD}$, and genetic analyses of $\mathrm{AD}$ based on a knowledge base.

\section{Diagnosis and Prognosis}

In earlier times, AI algorithms were used to make predictions for the diagnosis and prognosis of AD by analyzing patient genetic information. Takasaki et al. published two papers in 2008 and 2009 that studied centenarians and other patients in Japan with AD and Parkinson's disease.

In the first paper, they used a radial basis function (RBF) network to analyze mitochondrial single nucleotide polymorphisms (mtSNPs) at specific locations in mitochondrial DNA and found that different types of subjects have unique mtSNPs. Japanese patients with AD are closely related to the G2a haplogroup. The second paper showed that in addition to the G2a haplogroup, Japanese AD patients were associated with $\mathrm{B} 4 \mathrm{c} 1$ and N9b1 haplogroups. The authors believe that this analysis method can be used for the preliminary diagnosis to predict the possibility of someone developing $\mathrm{AD}$ or several other diseases [99, 100]. In 2011, Wei et al. developed a model-averaged naïve Bayes (MANB) model that performs better than previous models in predicting LOAD patients with 312 to 318 SNPs in 1,411 patients. The area under the receiver operating characteristic curve (AUC) reached 0.72. In addition, the model performs better when trained and tested with high-dimensional genomic data. The results support that MANB can be used to predict $\mathrm{AD}$ from genome-wide data [101].

In a recent study, $\mathrm{Xu}$ et al. created a support vector machine (SVM) algorithm to analyze gene-encoded protein sequences instead of patient genotype data. The algorithm was tested with 279 AD-related protein sequence data and 1,463 non-AD-related data from the UniProt database, and the prediction accuracy reached $85.7 \%$. However, the weakness of this study is that it does not distinguish protein sequence information between early-onset familial AD and other types of AD [102]. Wang et al. also used the SVM classifier to analyze the microarray gene expression dataset from the NCBI GEO database (www.ncbi.nlm.nih.gov/geo) to generate a gene coexpression network to identify possible AD diagnostic biomarkers. They identified a cluster of 44 genes as potential biomarkers [103].

Varatharajah et al. developed a multivariate model based on machine learning algorithms (SVM, multiple kernel learning), which integrates demographics, biomarkers of cerebrospinal fluid (CSF), magnetic resonance imaging (MRI), positron emission tomography (PET), a psychological test score for cognition and cognitive resilience, and top AD-related genes that have been validated (including a total of 94 potential predictive factors) to identify patients with mild cognitive impairment (MCI) who will progress to AD within three years. By analyzing 135 participants from the ADNI, their prediction accuracy rate was an astonishing 93\% [104]. The above research shows that the analysis of genetic data based on machine learning methods to predict the prognosis and risk stratification of $\mathrm{AD}$ has certain value, but if combined with imaging data, its accuracy will be greatly improved.

\section{Analysis of genetic variations in $A D$}

Since the use of GWAS to explore the genetic variation of AD, very high-dimensional SNPs have been generated, and most of these SNPs are irrelevant to AD. A study 
published by $\mathrm{Wu}$ et al. in 2012 proposed an effective stratified sampling method using a decision tree and Breiman random forest generation method to classify SNPs into multiple groups from an AD case-control dataset containing 380,157 SNPs [105]. Nguyen et al. also recognized the value of the random forest algorithm for identifying genetic variation from the GWAS dataset. An improved random forest method with quality-based twostage sampling was tested on a Parkinson and an AD GWAS SNP dataset; the results showed that this method was effective in selecting subgroups of SNPs, and the performance was even better than traditional ones [106].

Hamed et al. applied SMV classifiers with different kernels to analyze the ADNI-1 dataset, and the results confirmed that the APOE, ABCA7, BIN1, CD2AP, CD33, $C L U, C R 1, M S 4 A 6 A$, and PICALM loci found in previous GWAS were highly correlated with AD [107]. To address the challenge of individual data privacy concerns in the collaborative studies of GWAS from different institutions, $\mathrm{Li}$ et al. developed a processing framework that performs a sparse learning method (lasso regression analysis) in a distributed manner. Their method could exclude irrelevant features and rank SNPs that affect AD through risk without any privacy issues. An empirical study of SNP datasets from three independent institutions identified some risk SNP-associated genes for the diagnosis of $\mathrm{AD}$, including APOE, CD69, and PHACTR3 [108]. To improve the accuracy of identifying AD risk variants in the GWAS dataset, Lancour et al. suggested incorporating previously known information about $\mathrm{AD}$ candidate genes into the analysis. They developed an SVM approach that integrated genetic and network scores by propagating GWAS risk scores in a protein-protein interaction network to prioritize specific candidate disease genes from the ADGC GWAS dataset. A number of new $\mathrm{AD}$ risk candidate genes were predicted using this combination method. The top ten genes included $C R 2$, SHARPIN, PTPN2, C4B, TUBB2B, EPS8, PSMC3, STRAS, HSPA2, and STUB1. Pathway analysis showed that the ranking genes based on this combination approach were significantly enriched in AD-related pathways, including immune response, aging and hippocampal development [109].

With the development of brain imaging technology, it has been found that certain changes in the brain structure and function can occur for years before the clinical diagnosis of $\mathrm{AD}$ [110]. Many researchers have analyzed the association between brain imaging changes and genetic variations, and this is called neuroimaging genetics. Since 2004, the ADNI project funded by the United States National Institutes of Health and pharmaceutical companies has tracked and collected clinical, imaging, genetic, and biochemical biomarker data for $\mathrm{AD}$ patients, and it has become one of the most important $\mathrm{AD}$ brain imaging data sharing resources.

Wang et al. conducted a study in 2012 that used the sparse multimodal multitasking learning method to analyze imaging and GWAS genetic data from the ADNI database to identify AD-sensitive biomarkers. They were able to predict disease status and identify a range of phenotypes and genetic biomarkers, the latter included APOE, DAPK1, ENTPD7, SORCS1, BIN1, PICALM, SORL1, LOC651924, PRNP, and IL1B [111]. Another group used a parallel version of the random forest algorithm to produce an $\mathrm{AD}$ risk gene ranking by associating GWAS data with multiple quantitative neuroimaging traits from the ADNI database. The top 10 genes within 10k bases of the top-risk SNPs include TOMM40, APOE, PICALM, PVRL2, NTNG2, NTM, SLC12A1, MEF2D, CD109, UNC5B, and DPYD [112].

Sparse canonical correlation analysis (SCCA) can be used to identify the multivariate associations between multiple SNPs and neuroimaging traits. Du et al. created two structural SCCA models to analyze the associations between genetic markers within the $A P O E$ gene and magnetic resonance imaging (MRI) and amyloid imaging data retrieved from the ADNI database. They found that the $A P O E \varepsilon 4$ allele rs 429358 was strongly associated with damage to the right hippocampus and amyloid burden in the frontal region [113, 114]. Hou et al. used a multitask learning model to perform regression analysis on SNP and MRI datasets of ADNI, and they found several risk genetic variants of $\mathrm{AD}$ related to $A P O E, B C R, N P C 2$, and RFTN1 genes [115].

Certain genes may play a tissue-specific role in the pathogenesis of $\mathrm{AD}$. The network-wide association study (NetWAS) method can apply machine learning algorithms to tissue-specific functional interaction networks to prioritize analyzing the results of GWAS. Song et al. used this method to analyze the ADNI GWAS dataset with the hippocampal volume as the phenotype and found that the protocadherin alpha gene cluster (PCDHA) may be a suspicious gene [116]. The above studies have studied the association between genetic variations and static neuroimaging phenotypes at a single time point without considering the dynamics of phenotypic changes. Hao et al. hypothesized that these changing phenotypes could explain the dynamic neurodegeneration process and proposed a "temporally constrained group sparse canonical correlation analysis framework" that was trained with time series data from the ADNI database. They also focused on SNPs near the APOE gene and found that this model could detect stronger associations than previous SCCA models, confirming that the loci rs 76692773 and rs2075649 were top ranking; however, the longitudinal method questioned 
the impact of the risk locus rs 429358 on the deterioration of $\mathrm{AD}$ [117].

Table 1. Genetic risk factors for AD revealed by AI analysis exclusively

\begin{tabular}{|c|c|}
\hline Genetic risk factors for $\mathrm{AD}$ & Biological processes [https://www.genecards.org/] \\
\hline ANKRD36C [118] & Ion channel inhibitor activity \\
\hline$B C R[115]$ & Protein tyrosine kinase activity, macrophage functions, \\
\hline$C 4 B$ [109] & Inflammation \\
\hline CACNA1C [118] & Voltage-dependent calcium channel \\
\hline CD109 [112] & Inflammation \\
\hline CD69 [108] & Inflammation \\
\hline CLCN3 [118] & Voltage-gated chloride channel \\
\hline CR2 [109] & Inflammation \\
\hline DAPK1 [111] & Apoptosis modulation and signaling \\
\hline DHRSX [118] & Autophagy \\
\hline DPYD [112] & Uracil and thymidine catabolism. \\
\hline ENTPD7 [111] & Oxidative stress and DNA damage \\
\hline EPS8 [109] & Regulator of axonal filopodia formation in neurons \\
\hline FGF14 [118] & Nervous system development \\
\hline GALNT18 [118] & O-linked oligosaccharide biosynthesis \\
\hline GFRA1 [118] & Glial cell line-derived neurotrophic factor receptor family \\
\hline HSPA2 [109] & Molecular chaperone implicated in a wide variety of cellular processes \\
\hline IL1B [111] & Inflammation \\
\hline ITGBL1 [118] & EGF-like protein family \\
\hline LOC101928478 [118] & Long intergenic non-protein coding RNA \\
\hline LOC442028 [118] & Long intergenic non-protein coding RNA \\
\hline$M A F 1[118]$ & $\begin{array}{l}\text { Repression of RNA polymerase III-mediated transcription in response to } \\
\text { changing nutritional, environmental and cellular stress conditions }\end{array}$ \\
\hline$M E F 2 D[112]$ & Cell growth, survival and apoptosis \\
\hline NIPAI [118] & $\begin{array}{l}\text { Magnesium transporter that may play a role in nervous system } \\
\text { development and maintenance. }\end{array}$ \\
\hline NPC2 [115] & Transport of cholesterol \\
\hline$N T M[112]$ & Neural cell adhesion molecule \\
\hline NTNG2 [112] & Neurite outgrowth of both axons and dendrites \\
\hline OR11H4 [118] & Odorant receptor \\
\hline PCDHA [116] & Cell surface proteins of neurons and synaptic junctions \\
\hline PHACTR3 [108] & Nuclear scaffold in proliferating cells \\
\hline PPA1 [118] & Respiratory electron transport and ATP synthesis \\
\hline PRNP [111] & Neuronal development and synaptic plasticity \\
\hline PTPN2 [109] & Cell growth, differentiation and mitotic cycle \\
\hline PVRL2 [112] & Cell junction organization and adherens junction \\
\hline RFTN1 [115] & Inflammation \\
\hline RIMS1 [118] & Synaptic vesicle exocytosis \\
\hline SHARPIN [109] & Inflammation \\
\hline SLC12A1 [112] & Sodium-potassium-chloride cotransporter \\
\hline SORCS1 [111] & Neuropeptide receptor activity \\
\hline SORCS2 [118] & Receptor for the precursor forms of NGF and BDNF \\
\hline STRAP [109] & kinase activity \\
\hline STUB1 [109] & Inflammation \\
\hline TUBB2B [109] & Isoform of tubulin \\
\hline UNC5B [112] & Axon guidance \\
\hline
\end{tabular}

WGS data provide a new impetus for revealing extremely rare mutations affecting AD risks that GWAS cannot determine. Yang et al. explored a WGS database retrieved from ADNI through the Lasso regression, which included 6 million valid SNPs, baseline volumes of entorhinal cortex and hippocampus and their volume changes within 24 months. The top genes associated with the risk SNPs for the baseline volume of entorhinal cortex and hippocampus were APOE, ANKRD36C, GALNT18, GPC6, LOC442028, MAF1, ORI1H4, PPA1, and RIMS1. The top genes associated with the risk SNPs for the volume changes of EC and Hip were BACE2, CACNAIC, 
CLCN3, DHRSX, FGF14, GFRA1, ITGBL1, LOC101928478, NIPA1, SORCS2, and VATIL. Many of them had never been reported and required further validation. There were also many SNPs whose associated genes could not be identified [118]. Yang et al. further expanded their research by using a novel two-level structured sparse regression model that introduced sparsities in both nucleotide-level and gene networks. The analysis of WGS SNP and neural image data from ADNI showed that this method could effectively predict the risk SNVs associated with AD risk genes [119]. From the results of the above 14 papers, we understand that using machine learning algorithms to analyze the SNP data of GWAS and WGS can detect new genes and SNVs that may be related to AD risk (Table 1).

\section{Analysis of the Gene Expression Profile in AD}

Genetic variations alone or in combination with environmental factors can alter gene expression profiles in brain cells, cause abnormalities in the metabolism of certain proteins, and ultimately lead to pathological changes in AD. Studying changes in gene expression levels in cells of the brain is helpful to discover key genes and pathways related to the pathogenesis of $\mathrm{AD}$, which may be targets for therapeutic intervention. Highthroughput microarray and RNA-sequencing (RNA-Seq) based on next-generation sequencing technology can create a detailed view of the transcriptome of cell or tissue samples. Due to the high dimensionality and complexity of the data, they are hindered from gaining significant information about the biological processes of a specific disease. As a result, many studies have shifted from traditional statistical methods to machine learning methods for data analysis, effectively revealing complex biological characteristics.

In 2011, Kong et al. developed two unsupervised machine learning algorithms (independent component analysis, ICA, and nonnegative matrix factorization, $\mathrm{NMF}$ ) to analyze the microarray dataset of the hippocampal gene expression of control and AD samples. They found that changes in expression levels of many genes in the hippocampus of AD patients were related to metal metabolism and inflammation [120]. Scheubert was able to find relevant genes by using a wrapper approach of genetic algorithm and support vector machine (GA/SVM), which performed more efficiently by finding sets of genes that are less repetitive and more significantly attributed to $\mathrm{AD}$. Through analyzing a dataset consisting of six different brain regions from $87 \mathrm{AD}$ patients and 74 healthy control samples, they identified some new candidate biomarkers for $\mathrm{AD}$, including LOC642711, PRKXP1, LOC283345, SST and LY6H [121]. Panigrahi et al. applied an integrative systems biology approach to identify candidate genes and important biological processes among $\mathrm{AD}$ and aging. Supervised learning software and a self-organizing map implemented with an unsupervised artificial neural network were used to analyze three separate microarray datasets, and these included the CA1 region of the hippocampus, frontal lobe and blood mononuclear cells from $\mathrm{AD}$ and aging patients. Ten major classes of transcription factors and unique miRNA targets were identified as regulatory processes for $\mathrm{AD}$ in this study [122]. As the number of available microarray databases increases, many studies strive to find more significant genes using different methods or algorithms, such as the random forest method used by Nishiwaki et al. and the two-stage classifier consisting of relevance vector machine (RVM), SVM, random forest and extreme learning machine (ELM) classifiers developed by Miao et al. These methods both identified some candidate genes related to $\mathrm{AD}[123,124]$.

$\mathrm{Li}$ et al. studied the relationship of gene expression changes in blood and brain tissues by analyzing four blood and one brain tissue gene expression dataset. They found that more than $77 \%$ of genes have the same regulatory direction in different tissues and disease states. SVM, random forest and logistic ridge regression (RR) models showed that mitochondrial dysfunction, the NF-kappa B signaling pathway and iNOS signaling were important dysregulation pathways in the pathogenesis of $\mathrm{AD}$ [125]. It is currently known that controlling transcription through microRNA molecules is a key process in the development of late-onset AD. Armananzas et al. proposed a new method to integrate gene expression data and sequence predictions with a machine learning method. They analyzed two microRNAs and two gene expression datasets in temporal lobe samples and found some previously unreported the regulation of AD-related microRNAs, including miR-106a, miR-504, and miR$142-3 p$ [126].

Recent studies began using a more complicated approach, and many of these researchers believe that unconventional and complicated algorithms should be used to find any additional genes that play a role in $\mathrm{AD}$. Martinez-Ballesteros et al. combined decision tree classifiers, quantitative rules and hierarchical clustering methods and completed training on multiple carefully prepared gene expression datasets. However, they also considered additional sources, such as a repository of already relevant $\mathrm{AD}$ genes, gene ontology, and a literature review or expert knowledge, to validate their results. They found that the expression of 90 genes in patients with $\mathrm{AD}$ were significantly different from that of controls [127].

RNA-Seq uses next-generation sequencing technology to check the presence and quantity of all RNA in a sample, including alternative gene-splicing transcripts, posttranscriptional modifications, gene 
fusions, mutations, miRNA, tRNA, and ribosome profiles. In comparison, the weaknesses of hybrid-based microarrays are that they can only detect predesigned sequences and that the quantitative range is relatively narrow [128, 129]. Mukherjee et al. proposed an iterative multiview classifier using the logistic regression method. They used this classifier to analyze an RNA-seq dataset of 2,114 samples from seven different brain regions of 1,100 patients to identify potential AD risk (driver) genes. They found that the highest ranked genes contained several genes closely related to $\mathrm{AD}$, consistent with previous reports and that the results of the RNA-Seq data could be well verified by the GWAS data. Enrichment analysis found that in addition to well-known processes (such as immune response and amyloid processing), there are other processes (such as endocytosis, scavenger receptor activity and peptidase activity) that could lead to a new understanding of the mechanism of $\mathrm{AD}$ development [130]. Luo et al. conducted a small sample study using logistic regression classifiers by combining RNA-Seq data, a database from Online Mendelian Inheritance in Man (OMIM, www.omim.org/), and protein-protein interaction networks. The RNA-Seq dataset contains 9 AD subjects and 8 control subjects (GSE53697). They found that candidate $\mathrm{AD}$ genes were enriched in seven AD-related pathways, which included the NOD-like receptor signaling pathway, neurotrophin signaling pathway, and GnRH signaling pathway [131]. In summary, the 10 studies presented in this section show that the use of machine learning to analyze transcriptomes generated by microarrays and RNA-Seq can help discover genes and pathways that play important roles in the pathogenesis of AD.

\section{Gene-gene Interaction in $A D$}

As mentioned above, gene-gene interactions have significant roles in the pathogenesis of AD. Machine learning algorithms have been used in studies of SNP epistatic interactions, transcript interaction networks and metabolic pathways. In 2011, Jiang et al. created a combinatorial epistasis learning method with a Bayesian network. They evaluated the performance of this method with different parameters on simulated datasets and a real Alzheimer's GWAS dataset, and the results showed that this method is feasible [132]. Later, Jiang et al. combined Bayesian network and information gain algorithms to further improve the method. They analyzed a GWAS LOAD dataset that included 859 AD and 552 control cases. The results not only were consistent with previous reports but also indicated new interactions, i.e., APOE / GAB2 interactions involving more loci [133]. Han et al. also used a Bayesian network-based method to detect epistatic interactions from the same GWAS LOAD dataset as Jiang et al. They found two SNPs (rs1931565 and rs4505578), and their interactions with APOE might increase the risk of LOAD [134].

Granados et al. first used a multidimensional dimensionality reduction (MDR) algorithm to perform epistasis analysis on 12 AD-related SNPs. The dataset used was composed of 196 AD cases and 92 controls [135]. Zieselman et al. also recognized the value of MDR, and they used a quantitative multifactor dimensionality reduction (QMDR) method to analyze the SNP-SNP interactions on the GWAS LOAD dataset from ADNI. They found statistically significant synergistic interactions between several SNPs, but the results were not repeated in another independent dataset [136]. To address the combinatorial explosion problem of largescale GWAS datasets, Moore et al. introduced a method that combined expert knowledge and MDR methods to examine high-order gene-gene interactions. Expert knowledge from databases (such as gene ontology) or literature sources (such as PubMed) was used to filter gene datasets before the analysis. They applied this method to the GWAS dataset from ADNI and identified a set of interacting genes related to AD [137].

Another machine learning algorithm, iterative sure independence screening (SIS), can analyze very large datasets with more predictors than observations. An interaction analysis was performed by Hibar et al., which screened all possible SNP-SNP interactions that affected regional brain volumes from 534,033 SNPs in a GWAS dataset from ADNI. They found a significant SNP-SNP interaction between rs 1345203 (probably related to histone acetylation) and rs 1213205 (probably related to DNase I cleavage), which could explain $1.9 \%$ of the changes in the temporal lobe volume [138].

There are also many studies using machine learning methods to analyze transcript interaction networks. In an earlier study, Armananzas et al. used ensemble Bayesian network classifiers to build transcript interaction networks based on transcript profiling from entorhinal cortex and dentate gyrus samples in six AD and six control cases in 2012. Studies have found that some key transcripts in the network, such as S100A10, RPS3A, MED8, may have an important significance for the pathogenesis of AD [139]. The combinatorial optimization-based machine learning algorithm proposed by Ponzoni et al. was used to analyze the functional interconnection of two gene expression datasets in $\mathrm{AD}$ and control brain tissue. This approach provided both a global view of interconnections between different functional blocks and a specific molecular network of interest. The previously unreported ADrelated pathways obtained by this method included the citrate cycle, pyruvate metabolism, MAPK signaling, peroxisome, VEGF signaling, focal adhesion, 
aldosterone-regulated sodium reabsorption, carbohydrate digestion and absorption [140].

Zafeiris et al. designed an integrated artificial neural network (ANN) pipeline for biomarker discovery and verification in $A D$. By analyzing a gene expression microarray dataset (E-GEOD-48350) consisting of no less than 80 cases and at least four brain region samples, they generated a large and complex interaction dataset consisting of 500 gene probes and 1,000 predicted interactions, which could be used as a reference system to further examine genes of interest. Driver analysis produced a list of the most influential and most influenced genes that may be the source of imbalances in the metabolic system and therefore the most likely driver and treatment target for the disease [141]. Similar to the study by Zafeiris et al., Park et al. proposed a random forestbased algorithm to classify important gene-gene interactions. They tested the trained algorithm on an $\mathrm{AD}$ gene expression dataset (GSE15222). As a result, 3,366 $\mathrm{AD}$-associated gene-gene interactions were identified, and functional enrichment analysis showed that several AD-related pathways were significantly enriched [142].

Maj et al. took a different approach to study potential biological associations in different tissues with AD. They first applied a tissue-specific gene expression prediction model to predict the gene expression profiles of 42 nongender-specific tissues based on the genotypes of 808 samples from GWAS datasets of ADNI, which included controls, mild cognitive impairment subjects and $\mathrm{AD}$ patients. Then, the association between the AD cognitive decline and predicted tissue-specific gene expression was analyzed by different supervised and unsupervised machine learning methods. The advantage of using predicted transcriptome data is that it only reflects the role of genetic components and avoids environmental influences. Since epistatic interactions play a major role in the regulation of biochemical pathways, this study focused only on the analysis of regulatory networks, not univariate analyses. The results suggested that the inflammatory and regulatory processes in gut-brainrelated tissues had a potential effect on the cognitive decline [143].

Machine learning has also been used in the study of metabolic pathways. Coppede et al. were skeptical about the impairments in folate metabolism potentially being a risk for $\mathrm{AD}$ and used ANN to analyze 30 genetic and biochemical variables related to folate metabolism on a dataset that included 40 LOAD cases and 40 matching controls. Upon analyzing the results, they created a semantic connection map that could show complex biological associations between variables to differentiate $\mathrm{AD}$ cases from controls. The study found that certain variables (such as the TYMS and DNMT3B genotypes) may play an important role when considering the interaction of multiple variables in this pathway [144]. From the above 13 studies, it can be concluded that machine learning has significant advantages in analyzing and mapping complex networks of genetic and metabolic interactions.

\section{Genetic Analysis Based on a Knowledge base}

Most studies that used machine learning to decipher the pathogenesis of $\mathrm{AD}$ analyzed genetic or other medical information (such as brain imaging) from various original AD databases. However, there are few studies looking for alternative methods to help this development. These studies used AI technology to identify genes associated with $\mathrm{AD}$ risk by analyzing an established biological knowledge base.

Jamal et al. tried to find AD susceptible genes by employing eleven machine learning algorithms to analyze several open-source knowledge bases. The integrated topological properties of the AD-related genes were extracted from the protein-protein interaction networks (OPID, STRING, MINT, BIND and InTAct databases), sequence features (UniProt database) and functional annotations (DAVID and two additional Swiss-Prot functional annotation terms). They also used molecular docking methods to screen interactions between known drugs for $\mathrm{AD}$ and newly acquired $\mathrm{AD}$-related proteins [145]. In addition, Huang et al. used an SVM method to integrate the information from an $\mathrm{AD}$ gene knowledge base (AlzGene) and the brain-specific gene network data from GIANT, and then they analyzed more than 20,000 genes in a catalog of human genes and genetic disorders (OMIM). The candidate gene list of 832 genes generated in this study might provide a comprehensive reference for $\mathrm{AD}$ gene research [146]. Text mining tools can facilitate the literature search process. Singhal et al. proposed a machine learning method that could automatically extract disease-gene-variation information from biomedical literature. They extracted the above information about ten important diseases, including $\mathrm{AD}$, from all PubMed abstracts. After a comparison study with the UniProt knowledge base, the author believed that the method has practical value [147]. From this, we know that the integrated analysis of the knowledge base can provide some important research clues.

\section{Perspective for the Future}

The computing power and capabilities of developing technology increase exponentially every year. These new technologies have enabled the analysis of complex biological processes and diseases with extraordinary size and numerous dimensions. Especially for complex diseases, including $\mathrm{AD}$, analysis in a single or few 
dimensions prevents us from capturing the exact causes and factors associated with these diseases. Thus, efficient but complicated methods must be employed to combine multiple data types to pinpoint specific factors of a disease [16]. To the best of our knowledge, research papers on the genetics of $\mathrm{AD}$ using $\mathrm{AI}$ methods have only appeared in the last ten years, and many of them have focused on the exploration of research methods. In recent years, with the continuous increase in available public databases and the improvement of computer capabilities, research papers in this area have gradually increased. However, in general, the databases used for AI research are relatively limited, and few studies have applied a comprehensive analysis at multiple levels of genes, proteins, metabolism, and environmental factors. The conclusions of most studies are only to provide a reference for further research. Few studies have carried out a biological verification of the findings or proposed a verification scheme; in addition, few studies have proposed a theoretical framework for the pathogenesis of $\mathrm{AD}$ based on the results obtained [30, 58]. Regarding the use of AI technology to study the genetic factors of $\mathrm{AD}$ and the pathogenesis of $\mathrm{AD}$, current challenges, possible solutions and future development directions are discussed below.

(1) High quality, comprehensive, large sample size, and data sharing resources: the quality of the original data resources is the basic condition for obtaining correct results; comprehensive, large-sample data resources can improve researchers' ability to spot weak factors; in addition, shared data resources can provide opportunities for more research teams to participate in $\mathrm{AD}$ research. The ANDI database is a very successful example of this. Other large shareable databases have been listed above and include ADGC, ADSP, EMBL-EBI, GAAIN, NCBI, NIAGADS, etc. However, the majority of these data come from only developed countries and do not include major populations in developing countries. It is believed that future international cooperation will also promote the improvement and development of these shared databases [98]. For genome and gene expression databases, WGS and RNA-Seq based on next-generation sequencing can detect nonpredesigned sequences; thus, these have more advantages than microarray-based databases. We believe that such databases will play a more important role in the future. The organism as a whole is a complex system of genes, proteins, cells, individuals and environmental factors (including various physiological and biochemical conditions of the internal and external environments, including living conditions, lifestyle, social psychology, etc.) at different levels. Establishing and improving the dynamic database of the corresponding genome, epigenome, transcriptome, proteome, metabolome, microbiome and other internal and external environmental factors are the future development directions. Currently, the ability to collect data from internal and external environmental factors is still very limited. It is believed that with the development of nanotechnology [148], wearable devices [149], the Internet of things [150], smartphone applications [151], and other technologies [152], these dynamic databases will also be established. In addition, knowledge bases, including AlzGene and UniProt knowledge base, also play a vital role.

(2) Multilevel system biological analysis strategy: $\mathrm{AD}$ is the result of the complex interactions between genes and the environment. Therefore, the study of its pathogenesis needs to include the interaction between the genome and environmental factors, as well as the epigenome, transcriptome, proteome, metabolome, and microbiome, in related cells and tissues. A section in this article specifically discusses the study of genes and gene interactions by AI technology. Indeed, these studies have also found many meaningful interactions for the pathogenesis of AD. Systems biology is an interdisciplinary research area that uses holistic methods to analyze complex interactions in biological systems through mathematical models [153]. We believe that this analysis strategy of systems biology combining various aspects of information will continue to progress with incredible advances in computing power, new AI algorithms and availability of data. Ultimately, a comprehensive AI analysis system can be established, which will be of great significance for a thorough understanding of the pathogenesis of complex diseases such as $\mathrm{AD}[16,154]$.

(3) Theoretical summary and verification of AI analysis results: Conclusions from most of the studies presented in this article only indicate that certain genes or pathways may be related to the pathogenesis of $\mathrm{AD}$, and these only provide a reference for further research. Can the study of the pathogenesis of AD using AI technology only reach this stage? Of course not. The goal of theoretical research is to be able to propose a verifiable model of the research object. For simple objects, such as the motion of objects, this theoretical model can be expressed using very simple mathematical formulas, but for complex diseases, such as $\mathrm{AD}$, due to the interaction of many factors, it can be speculated that this theoretical model must be a complex model built on a computer program [155]. Can the establishment of such a theoretical model only depend on the creativity of human thinking? Can AI help us build these complex models? We have reason to believe that AI technology can help. In fact, although computational creativity is still in its infancy, it is also one of the important branches of the rapid development of AI. It is committed to making computers have the ability to play independent creators or cocreators. Its application researches include literature and 
art creation (such as stories, poetry, paintings, musical works, games), problem solving, and system design, etc. $[156,157]$. Some form of machine learning has been used in almost all the studies presented in this article. Although this method has indeed reached a certain level of practicality, machine learning is only a small part of AI technology. We boldly speculate that a more comprehensive AI technology including computational creativity and machine learning can not only help us make accurate diagnosis and prediction, but also help us analyze the research results, propose new hypotheses or theoretical models, design feasible verification schemes, and new intervention protocols. AI technology will bring humanity into a new era $[56,71,158]$.

\section{Concluding Remarks}

Most cases of $\mathrm{AD}$ are the result of a complex interaction of multiple genes and environmental factors, and traditional genetic analysis methods were successful in discovering many of the significant genes and factors for the pathogenesis of $\mathrm{AD}$. In recent years, with the development of large databases such as GWAS, gene expression array, WGS, and RNA-Seq, the analysis and exploration of data by conventional statistical methods have shown certain limitations. AI technology (machine learning algorithms) has been applied to the analysis of genetic variations, gene expression profiles and genegene interactions of $\mathrm{AD}$ in the past 10 years and has produced some meaningful results. Although it is still at a relatively preliminary stage, we believe that with the continuous improvement of high-quality, comprehensive, large sample size, data sharing resources, applying multilevel system biological analysis strategies, and incredible advances in computing power, a comprehensive analysis system can eventually be established and help to fully understand the pathogenesis of AD. In the future, computational creativity may play a role in building and verifying a theory model and designing new intervention protocols for $\mathrm{AD}$.

\section{Acknowledgements}

This study was supported by the Washington Institute for Health Sciences grant (G20190710).

\section{Conflict of Interest}

No conflicts of interest are declared.

\section{References}

Puri B, Hall A and Ho R, editors. Revision notes in psychiatry (3rd ed.). New York: CRC Press; 2013.
Sallim AB, Sayampanathan AA, Cuttilan A, Ho R (2015). Prevalence of Mental Health Disorders among Caregivers of Patients with Alzheimer Disease. J Am Med Dir Assoc, 16:1034-1041.

[3] United Nations, Department of Economic and Social Affairs, Population Division. World Population Ageing. New York: The United Nations; 2015 (ST/ESA/SER.A/390)

[4] Prince M, Comas-Herrera A, Knapp M, Guerchet M, Karagiannidou M. World Alzheimer Report 2016: Improving healthcare for people living with dementia. Coverage, quality and costs now and in the future. London: Alzheimer's Disease International (ADI); 2016.

[5] Alzheimer's Association (2019). 2019 Alzheimer's Disease Facts and figures. Alzheimers Dement, 15:321387.

[6] Piaceri I, Nacmias B, Sorbi S (2013). Genetics of familial and sporadic Alzheimer's disease. Front Biosci (Elite Ed), 5:167-177.

[7] Ng A, Tam WW, Zhang MW, Ho CS, Husain SF, McIntyre RS, et al. (2018). IL-1 $\beta$, IL-6, TNF- $\alpha$ and CRP in Elderly Patients with Depression or Alzheimer's disease: Systematic Review and Meta-Analysis. Sci Rep, 8:12050.

[8] Ng TKS, Ho CSH, Tam WWS, Kua EH, Ho RC (2019). Decreased Serum Brain-Derived Neurotrophic Factor (BDNF) Levels in Patients with Alzheimer's Disease (AD): A Systematic Review and Meta-Analysis. Int J Mol Sci, 20:257.

[9] Gatz M, Reynolds CA, Fratiglioni L, Johansson B, Mortimer JA, Berg S, et al. (2006). Role of genes and environments for explaining Alzheimer disease. Arch Gen Psychiatry, 63:168-174.

[10] Ballard C, Gauthier S, Corbett A, Brayne C, Aarsland D, Jones E (2011). Alzheimer's disease. Lancet, 377:1019-1031.

[11] Freudenberg-Hua Y, Li W, Davies P (2018). The Role of Genetics in Advancing Precision Medicine for Alzheimer's Disease-A Narrative Review. Front Med (Lausanne), 5:108.

[12] Bi C, Bi S, Li B (2019). Processing of Mutant $\beta$ Amyloid Precursor Protein and the Clinicopathological Features of Familial Alzheimer's Disease. Aging Dis. 10:383-403.

[13] Lane CA, Hardy J, Schott JM (2018). Alzheimer's disease. Eur J Neurol, 25:59-70.

[14] Frazer KA, Murray SS, Schork NJ, Topol EJ (2009). Human genetic variation and its contribution to complex traits. Nat Rev Genet, 10:241-251.

[15] Mazzocchi F (2008). Complexity in biology. Exceeding the limits of reductionism and determinism using complexity theory. EMBO Rep, 9:10-14.

[16] Zitnik M, Nguyen F, Wang B, Leskovec J, Goldenberg A, Hoffman MM (2019). Machine Learning for Integrating Data in Biology and Medicine: Principles, Practice, and Opportunities. Inf Fusion, 50:71-91.

[17] Rajkomar A, Dean J, Kohane I (2019). Machine Learning in Medicine. N Engl J Med, 380:1347-1358. 
[18] Webb S (2018). Deep learning for biology. Nature, 554:555-557.

[19] Tran BX, Vu GT, Ha GH, Vuong QH, Ho MT, Vuong TT, et al. (2019). Global Evolution of Research in Artificial Intelligence in Health and Medicine: A Bibliometric Study. J Clin Med, 8:360.

[20] Lee Y, Ragguett RM, Mansur RB, Boutilier JJ, Rosenblat JD, Trevizol A, et al. (2018). Applications of machine learning algorithms to predict therapeutic outcomes in depression: A meta-analysis and systematic review. J Affect Disord, 241:519-532.

[21] Philips A. Brey (2000). Theories of Technology as Extension of Human Faculties. In: Mitcham C, editors. Metaphysics, Epistemology and Technologytable. Londen: Elsevier/JAI Press, 59-78.

[22] Russell SJ, Norvig P, editors. Artificial Intelligence: A Modern Approach (3rd ed.). Upper Saddle River: Prentice Hall; 2009

[23] Legg S, Hutter M (2007). A Collection of Definitions of Intelligence. Cornell University Library, arXiv:0706.3639

[24] Ertel W. Introduction to Artificial Intelligence. 2nd ed. Cham: Springer International Publishing; 2017.

[25] Preparing for the Future of Artificial Intelligence. Washington, D.C: Executive Office of the President; 2016.

[26] Wu J, Zhao Y (2019). Machine learning technology in the application of genome analysis: A systematic review. Gene, 705:149-156.

[27] Noorbakhsh-Sabet N, Zand R, Zhang Y, Abedi V (2019). Artificial Intelligence Transforms the Future of Health Care. Am J Med, 132:795-801.

[28] Miotto R, Wang F, Wang S, Jiang X, Dudley JT (2018). Deep learning for healthcare: review, opportunities and challenges. Brief Bioinform, 19:1236-1246.

[29] Fogel AL, Kvedar JC (2018). Artificial intelligence powers digital medicine. NPJ Digit Med, 1:5.

[30] Topol EJ (2019). High-performance medicine: the convergence of human and artificial intelligence. Nat Med, 25:44-56.

[31] Shah P, Kendall F, Khozin S, Goosen R, Hu J, Laramie $\mathrm{J}$, et al. (2019). Artificial intelligence and machine learning in clinical development: a translational perspective. NPJ Digit Med, 2:69.

[32] McCarthy J, Minsky M, Rochester N, Shannon C (2006). A proposal for the Dartmouth Summer Research Project on artificial intelligence, August 31, 1955. AI Magazine, 27:12-14.

[33] Miller RA (1994). Medical diagnostic decision support systems - past, present, and future: a threaded bibliography and brief commentary. J Am Med Inform Assoc, 1:8-27.

[34] Hosny A, Parmar C, Quackenbush J, Schwartz LH, Aerts HJWL (2018). Artificial intelligence in radiology. Nat Rev Cancer, 18:500-510.

[35] Bera K, Schalper KA, Rimm DL, Velcheti V, Madabhushi A (2019). Artificial intelligence in digital pathology - new tools for diagnosis and precision oncology. Nat Rev Clin Oncol, 16:703-715.
[36] Hogarty DT, Su JC, Phan K, Attia M, Hossny M, Nahavandi S, et al. (2019). Artificial Intelligence in Dermatology-Where We Are and the Way to the Future: A Review. Am J Clin Dermatol, Epub ahead of print.

[37] Von der Emde L, Pfau M, Dysli C, Thiele S, Möller PT, Lindner M, et al. (2019). Artificial intelligence for morphology-based function prediction in neovascular age-related macular degeneration. Sci Rep, 9:11132.

[38] Johnson KW, Torres Soto J, Glicksberg BS, Shameer K, Miotto R, Ali M, et al. (2018). Artificial Intelligence in Cardiology. J Am Coll Cardiol, 71:2668-2679.

[39] Titano JJ, Badgeley M, Schefflein J, Pain M, Su A, Cai M, et al. (2018). Automated deep-neural-network surveillance of cranial images for acute neurologic events. Nat Med, 24:1337-1341.

[40] Rees CJ, Koo S (2019). Artificial intelligence - upping the game in gastrointestinal endoscopy? Nat Rev Gastroenterol Hepatol, 16:584-585.

[41] Hashimoto DA, Rosman G, Rus D, Meireles OR (2018). Artificial Intelligence in Surgery: Promises and Perils. Ann Surg, 268:70-76.

[42] Ding Y, Sohn JH, Kawczynski MG, Trivedi H, Harnish R, Jenkins NW, et al. (2019). A Deep Learning Model to Predict a Diagnosis of Alzheimer Disease by Using (18)F-FDG PET of the Brain. Radiology, 290:456-464.

[43] Guo J, Li B (2018). The Application of Medical Artificial Intelligence Technology in Rural Areas of Developing Countries. Health Equity, 2:174-181.

[44] Shaban-Nejad A, Michalowski M, Buckeridge DL (2018). Health intelligence: how artificial intelligence transforms population and personalized health. NPJ Digit Med, 1:53.

[45] Esteva A, Robicquet A, Ramsundar B, Kuleshov V, DePristo M, Chou K, et al. (2019). A guide to deep learning in healthcare. Nat Med, 25:24-29.

[46] Thiébaut R, Thiessard F, Section Editors for the IMIA Yearbook Section on Public Health and Epidemiology Informatics (2018). Artificial Intelligence in Public Health and Epidemiology. Yearb Med Inform, 27:207210.

[47] Zou J, Huss M, Abid A, Mohammadi P, Torkamani A, Telenti A (2019). A primer on deep learning in genomics. Nat Genet, 51:12-18.

[48] Eraslan G, Avsec Z, Gagneur J, Theis FJ (2019). Deep learning: new computational modelling techniques for genomics. Nat Rev Genet, 20:389-403.

[49] Holder LB, Haque MM, Skinner MK (2017). Machine learning for epigenetics and future medical applications. Epigenetics, 12:505-514.

[50] Telenti A, Lippert C, Chang PC, DePristo M (2018). Deep learning of genomic variation and regulatory network data. Hum Mol Genet, 27(R1):R63-R71.

[51] Cao C, Liu F, Tan H, Song D, Shu W, Li W, et al. (2018). Deep Learning and Its Applications in Biomedicine. Genomics Proteomics Bioinformatics, 16:17-32.

[52] Deng H, Jia Y, Zhang Y (2018). Protein structure prediction. Int J Mod Phys B, 32 ppi:1840009. 
[53] Nketia TA, Sailem H, Rohde G, Machiraju R, Rittscher J (2017). Analysis of live cell images: Methods, tools and opportunities. Methods, 115:65-79.

[54] Kan A (2017). Machine learning applications in cell image analysis. Immunol Cell Biol, 95:525-530.

[55] Schneider G (2018). Automating drug discovery. Nat Rev Drug Discov, 17:97-113.

[56] Paranjpe MD, Taubes A, Sirota M (2019). Insights into Computational Drug Repurposing for Neurodegenerative Disease. Trends Pharmacol Sci, 40:565-576.

[57] Harrer S, Shah P, Antony B, Hu J (2019). Artificial Intelligence for Clinical Trial Design. Trends Pharmacol Sci, 40:577-591.

[58] He J, Baxter SL, Xu J, Xu J, Zhou X, Zhang K (2019). The practical implementation of artificial intelligence technologies in medicine. Nat Med, 25:30-36.

[59] Ku CS, Loy EY, Salim A, Pawitan Y, Chia KS (2010). The discovery of human genetic variations and their use as disease markers: Past, present and future. J Hum Genet, 55:403-415.

[60] Maston GA, Evans SK, Green MR (2006). Transcriptional regulatory elements in the human genome. Annu Rev Genomics Hum Genet, 7:29-59.

[61] ENCODE Project Consortium (2012). An integrated encyclopedia of DNA elements in the human genome. Nature, 489:57-74.

[62] Fenoglio C, Scarpini E, Serpente M, Galimberti D (2018). Role of Genetics and Epigenetics in the Pathogenesis of Alzheimer's Disease and Frontotemporal Dementia. J Alzheimers Dis, 62:913932.

[63] Dawn Teare M, Barrett JH (2005). Genetic linkage studies. Lancet, 366:1036-1044.

[64] Lill CM, Bertram L (2011). Towards unveiling the genetics of neurodegenerative diseases. Semin Neurol, 31:531-541.

[65] Wiseman FK, Al-Janabi T, Hardy J, Karmiloff-Smith A, Nizetic D, Tybulewicz VL, et al. (2015). A genetic cause of Alzheimer disease: mechanistic insights from Down syndrome. Nat Rev Neurosci, 16:564-574.

[66] Kehoe P, Wavrant-De Vrieze F, Crook R, Wu WS, Holmans $\mathrm{P}$, et al. (1999). A full genome scan for late onset Alzheimer's disease. Hum Mol Genet. 1999 Feb;8(2):237-45.

[67] Wilkening S, Chen B, Bermejo JL, Canzian F (2009). Is there still a need for candidate gene approaches in the era of genome-wide association studies? Genomics, 93:415-419.

[68] Manolio TA (2010). Genomewide association studies and assessment of the risk of disease. N Engl J Med, 363:166-176.

[69] Lambert JC, Ibrahim-Verbaas CA, Harold D, Naj AC, Sims R, Bellenguez C, et al. (2013). Meta-analysis of 74,046 individuals identifies 11 new susceptibility loci for Alzheimer's disease. Nat Genet, 45:1452-1458.

[70] Kunkle BW, Grenier-Boley B, Sims R, Bis JC, Damotte $\mathrm{V}$, Naj AC, et al. (2019). Genetic meta-analysis of diagnosed Alzheimer's disease identifies new risk loci and implicates $\mathrm{A} \beta$, tau, immunity and lipid processing. Nat Genet, 51:414-430.

[71] Pimenova AA, Raj T, Goate AM (2018). Untangling Genetic Risk for Alzheimer's Disease. Biol Psychiatry, 83:300-310.

[72] Behjati S, Tarpey PS (2013). What is next generation sequencing? Arch Dis Child Educ Pract Ed, 98:236238.

[73] Patel D, Mez J, Vardarajan BN, Staley L, Chung J, Zhang X, et al. (2019). Association of Rare Coding Mutations With Alzheimer Disease and Other Dementias Among Adults of European Ancestry. JAMA Netw Open, 2:e191350.

[74] Pottier C, Hannequin D, Coutant S, Rovelet-Lecrux A, Wallon D, Rousseau S, et al. (2012). High frequency of potentially pathogenic SORL1 mutations in autosomal dominant early-onset Alzheimer disease. Mol Psychiatry, 17:875-879.

[75] Xu Y, Liu X, Shen J, Tian W, Fang R, Li B, et al. (2018). The Whole Exome Sequencing Clarifies the Genotype- Phenotype Correlations in Patients with Early-Onset Dementia. Aging Dis, 9:696-705.

[76] Beecham GW, Vardarajan B, Blue E, Bush W, Jaworski J, Barral S, et al. (2018). Rare genetic variation implicated in non-Hispanic white families with Alzheimer disease. Neurol Genet, 4:e286.

[77] Bis JC, Jian X, Kunkle BW, Chen Y, Hamilton-Nelson KL, Bush WS, et al. (2018). Whole exome sequencing study identifies novel rare and common Alzheimer'sAssociated variants involved in immune response and transcriptional regulation. Mol Psychiatry, Epub ahead of print.

[78] Blue EE, Bis JC, Dorschner MO, Tsuang DW, Barral SM, Beecham G, et al. (2018). Genetic Variation in Genes Underlying Diverse Dementias May Explain a Small Proportion of Cases in the Alzheimer's Disease Sequencing Project. Dement Geriatr Cogn Disord, 45:1-17.

[79] Blue EE, Yu CE, Thornton TA, Chapman NH, Kernfeld $\mathrm{E}$, Jiang N, et al. (2018). Variants regulating ZBTB4 are associated with age-at-onset of Alzheimer's disease. Genes Brain Behav, 17:e12429.

[80] Cruchaga C, Karch CM, Jin SC, Benitez BA, Cai Y, Guerreiro R, et al. (2014). Rare coding variants in the phospholipase D3 gene confer risk for Alzheimer's disease. Nature, 505:550-554.

[81] Kohli MA, Cukier HN, Hamilton-Nelson KL, Rolati S, Kunkle BW, Whitehead PL, et al. (2016). Segregation of a rare TTC3 variant in an extended family with lateonset Alzheimer disease. Neurol Genet, 2:e41.

[82] Jakobsdottir J, van der Lee SJ, Bis JC, Chouraki V, LiKroeger D, Yamamoto S, et al. (2016). Rare Functional Variant in TM2D3 is Associated with Late-Onset Alzheimer's Disease. PLoS Genet, 12:e1006327.

[83] Escott-Price V, Shoai M, Pither R, Williams J, Hardy J (2017). Polygenic score prediction captures nearly all common genetic risk for Alzheimer's disease. Neurobiol Aging, 49:214.e7-214.e11.

[84] Gusareva ES, Carrasquillo MM, Bellenguez C, Cuyvers E, Colon S, Graff-Radford NR, et al. (2014). Genome- 
wide association interaction analysis for Alzheimer's disease. Neurobiol Aging, 35:2436-2443.

[85] Raghavan N, Tosto G (2017). Genetics of Alzheimer's Disease: the Importance of Polygenic and Epistatic Components. Curr Neurol Neurosci Rep, 17:78.

[86] Goldman JS, Van Deerlin VM (2018). Alzheimer's Disease and Frontotemporal Dementia: The Current State of Genetics and Genetic Testing Since the Advent of Next-Generation Sequencing. Mol Diagn Ther, 22:505-513.

[87] Ng KST, Sia A, Ng MKW, Tan CTY, Chan HY, Tan $\mathrm{CH}$, et al. (2018). Effects of Horticultural Therapy on Asian Older Adults: A Randomized Controlled Trial. Int J Environ Res Public Health, 15:1705.

[88] Ho RC, Zhang MW, Ho CS, Pan F, Lu Y, Sharma VK (2014). Impact of 2013 south Asian haze crisis: study of physical and psychological symptoms and perceived dangerousness of pollution level. BMC Psychiatry, $14: 81$.

[89] Ho RC, Lam SY, Tan ES, Ng PM, Mak A (2009). Amnesia, political ambition, and canned tuna. Lancet, 373:352.

[90] Ho CSH, Ho RCM, Quek AML (2018). Chronic Manganese Toxicity Associated with Voltage-Gated Potassium Channel Complex Antibodies in a Relapsing Neuropsychiatric Disorder. Int J Environ Res Public Health, 15:783.

[91] Luck T, Riedel-Heller SG, Luppa M, Wiese B, Köhler M, Jessen F, et al. (2014). Apolipoprotein E epsilon 4 genotype and a physically active lifestyle in late life: analysis of gene-environment interaction for the risk of dementia and Alzheimer's disease dementia. Psychol Med, 44:1319-1329.

[92] Eid A, Mhatre I, Richardson JR (2019). Geneenvironment interactions in Alzheimer's disease: A potential path to precision medicine. Pharmacol Ther, 199:173-187.

[93] Kanherkar RR, Bhatia-Dey N, Csoka AB (2014). Epigenetics across the human lifespan. Front Cell Dev Biol, 2:49.

[94] Liu X, Jiao B, Shen L (2018). The Epigenetics of Alzheimer's Disease: Factors and Therapeutic Implications. Front Genet, 9:579.

[95] Ridge PG, Kauwe JSK (2018). Mitochondria and Alzheimer's Disease: the Role of Mitochondrial Genetic Variation. Curr Genet Med Rep, 6:1-10.

[96] Onyango IG, Dennis J, Khan SM (2016). Mitochondrial Dysfunction in Alzheimer's Disease and the Rationale for Bioenergetics Based Therapies. Aging Dis, 7:201214.

[97] Ashish N, Bhatt P, Toga AW (2016). Global Data Sharing in Alzheimer Disease Research. Alzheimer Dis Assoc Disord, 30):160-168.

[98] Toga AW (2018). Data Sharing in Alzheimer's Disease Research. US Neurol, 14:68-70

[99] Takasaki S (2008). Mitochondrial SNPs associated with Japanese centenarians, Alzheimer's patients, and Parkinson's patients. Comput Biol Chem, 32:332-337.
[100] Takasaki S (2009). Mitochondrial haplogroups associated with Japanese Alzheimer's patients. J Bioenerg Biomembr, 41:407-410.

[101] Wei W, Visweswaran S, Cooper GF (2011). The application of naive Bayes model averaging to predict Alzheimer's disease from genome-wide data. J Am Med Inform Assoc, 18:370-375.

[102] Xu L, Liang G, Liao C, Chen GD, Chang CC (2018). An Efficient Classifier for Alzheimer's Disease Genes Identification. Molecules, 23:E3140.

[103] Wang L, Liu ZP (2019). Detecting Diagnostic Biomarkers of Alzheimer's Disease by Integrating Gene Expression Data in Six Brain Regions. Front Genet, 10:157.

[104] Varatharajah Y, Ramanan VK, Iyer R, Vemuri P; Alzheimer's Disease Neuroimaging Initiative (2019). Predicting Short-term MCI-to-AD Progression Using Imaging, CSF, Genetic Factors, Cognitive Resilience, and Demographics. Sci Rep, 9:2235.

[105] Wu Q, Ye Y, Liu Y, Ng MK (2012). SNP selection and classification of genome-wide SNP data using stratified sampling random forests. IEEE Trans Nanobioscience, 11:216-227.

[106] Nguyen TT, Huang J, Wu Q, Nguyen T, Li M (2015). Genome-wide association data classification and SNPs selection using two-stage quality-based Random Forests. BMC Genomics, 16 Suppl 2:S5.

[107] El-Hamid MMA, Omar YMK, and Mabrouk MS (2016). Identifying genetic biomarkers associated to Alzheimer's disease using Support Vector Machine. 8th Cairo International Biomedical Engineering Conference. Cairo; 5-9.

[108] Li Q, Yang T, Zhan L, Hibar DP, Jahanshad N, Wang Y, et al. (2016). Large-scale Collaborative Imaging Genetics Studies of Risk Genetic Factors for Alzheimer's Disease Across Multiple Institutions. Cornell University Library, arXiv:1608.07251

[109] Lancour D, Naj A, Mayeux R, Haines JL, PericakVance MA, Schellenberg GD, et al. (2018). One for all and all for One: Improving replication of genetic studies through network diffusion. PLoS Genet, 14:e1007306.

[110] Twamley EW, Ropacki SA, Bondi MW (2006). Neuropsychological and neuroimaging changes in preclinical Alzheimer's disease. J Int Neuropsychol Soc, 12:707-735.

[111] Wang H, Nie F, Huang H, Risacher SL, Saykin AJ, Shen L, et al. (2012). Identifying disease sensitive and quantitative trait-relevant biomarkers from multidimensional heterogeneous imaging genetics data via sparse multimodal multitask learning. Bioinformatics, 28:i127-136.

[112] Wang Y, Goh W, Wong L, Montana G; Alzheimer's Disease Neuroimaging Initiative (2013). Random forests on Hadoop for genome-wide association studies of multivariate neuroimaging phenotypes. BMC Bioinformatics, 14 Suppl 16:S6.

[113] Du L, Jingwen Y, Kim S, Risacher SL, Huang H, Inlow M, et al. (2014). A novel structure-aware sparse learning algorithm for brain imaging genetics. Med Image Comput Comput Assist Interv, 17(Pt 3):329-336. 
[114] Du L, Huang H, Yan J, Kim S, Risacher S, Inlow M, et al. (2016). Structured sparse CCA for brain imaging genetics via graph OSCAR. BMC Syst Biol, 10 Suppl 3:68.

[115] Huo Z, Shen D, Huang H (2018). Genotype-phenotype association study via new multi-task learning model. Pac Symp Biocomput, 23:353-364.

[116] Song A, Yan J, Kim S, Risacher SL, Wong AK, Saykin AJ, et al. (2016). Network-based analysis of genetic variants associated with hippocampal volume in Alzheimer's disease: a study of ADNI cohorts. BioData Min, 9:3.

[117] Hao X, Li C, Yan J, Yao X, Risacher SL, Saykin AJ, et al. (2017). Identification of associations between genotypes and longitudinal phenotypes via temporallyconstrained group sparse canonical correlation analysis. Bioinformatics. 33:i341-i349.

[118] Yang T, Wang J, Sun Q, Hibar DP, Jahanshad N, Liu L, et al. (2015). Detecting Genetic Risk Factors for Alzheimer's Disease in Whole Genome Sequence Data via Lasso Screening. Proc IEEE Int Symp Biomed Imaging, 2015:985-989.

[119] Yang T, Thompson P, Zhao S, Ye J (2017). Identifying Genetic Risk Factors via Sparse Group Lasso with Group Graph Structure. Cornell University Library, arXiv:1709.03645.

[120] Kong W, Mou X, Hu X (2011). Exploring matrix factorization techniques for significant genes identification of Alzheimer's disease microarray gene expression data. BMC Bioinformatics. 12 Suppl 5:S7.

[121] Scheubert L, Luštrek M, Schmidt R, Repsilber D, Fuellen G (2012). Tissue-based Alzheimer gene expression markers-comparison of multiple machine learning approaches and investigation of redundancy in small biomarker sets. BMC Bioinformatics, 13:266.

[122] Panigrahi PP, Singh TR (2013). Computational studies on Alzheimer's disease associated pathways and regulatory patterns using microarray gene expression and network data: revealed association with aging and other diseases. J Theor Biol, 334:109-121.

[123] Nishiwaki K, Kanamori K, Ohwada H (2016). Finding a Disease-Related Gene from Microarray Data using Random Forest. IEEE 15th International Conference on Cognitive Informatics \& Cognitive Computing. IEEE Xplore: 16693156.1

[124] Miao Y, Jiang H, Liu H, Yao YD (2017). An Alzheimers disease related genes identification method based on multiple classifier integration. Comput Methods Programs Biomed, 150:107-115.

[125] Li X, Wang H, Long J, Pan G, He T, Anichtchik O, et al. (2018). Systematic Analysis and Biomarker Study for Alzheimer's Disease. Sci Rep, 8:17394.

[126] Armañanzas R (2018). Revealing post-transcriptional microRNA-mRNA regulations in Alzheimer's disease through ensemble graphs. BMC Genomics, 19(Suppl 7):668.

[127] Martínez-Ballesteros M, García-Heredia JM, Nepomuceno-Chamorro IA, Riquelme-Santos JC (2017). Machine learning techniques to discover genes with potential prognosis role in Alzheimer's disease using different biological sources. Information Fusion, 36: 114-129.

[128] Wang Z, Gerstein M, Snyder M (2009). RNA-Seq: a revolutionary tool for transcriptomics. Nat Rev Genet, 10:57-63.

[129] Kukurba KR, Montgomery SB (2015). RNA Sequencing and Analysis. Cold Spring Harb Protoc, 2015:951-969.

[130] Mukherjee S, Perumal TM, Daily K, Sieberts SK, Omberg L, Preuss C, et al. (2019). Identifying and ranking potential driver genes of Alzheimer's disease using multiview evidence aggregation. Bioinformatics, 35:i568-i576.

[131] Luo P, Tian LP, Ruan J, Wu FX (2019). Disease Gene Prediction by Integrating PPI Networks, Clinical RNASeq Data and OMIM Data. IEEE/ACM Trans Comput Biol Bioinform, 16:222-232.

[132] Jiang X, Neapolitan RE, Barmada MM, Visweswaran S (2011). Learning genetic epistasis using Bayesian network scoring criteria. BMC Bioinformatics, 12:89.

[133] Jiang X, Jao J, Neapolitan R (2015). Learning Predictive Interactions Using Information Gain and Bayesian Network Scoring. PLoS One, 10:e0143247.

[134] Han B, Chen XW, Talebizadeh Z, Xu H (2012). Genetic studies of complex human diseases: characterizing SNP-disease associations using Bayesian networks. BMC Syst Biol, 6 Suppl 3:S14.

[135] Granados EAO, Vásquez LFN, Granados HA (2013). Characterizing genetic interactions using a machine learning approach in Colombian patients with Alzheimer's disease. 2013 IEEE International Conference on Bioinformatics and Biomedicine. IEEE Xplore: 14079653.

[136] Zieselman AL, Fisher JM, Hu T, Andrews PC, Greene CS, Shen L, et al. (2014). Computational genetics analysis of grey matter density in Alzheimer's disease. BioData Min, 7:17.

[137] Moore JH, Andrews PC, Olson RS, Carlson SE, Larock CR, Bulhoes MJ, et al. (2017). Grid-based stochastic search for hierarchical gene-gene interactions in population-based genetic studies of common human diseases. BioData Min, 10:19.

[138] Hibar DP, Stein JL, Jahanshad N, Kohannim O, Hua X, Toga AW, et al. (2015). Genome-wide interaction analysis reveals replicated epistatic effects on brain structure. Neurobiol Aging, 36 Suppl 1:S151-8.

[139] Armañanzas R, Larrañaga P, Bielza C (2012). Ensemble transcript interaction networks: a case study on Alzheimer's disease. Comput Methods Programs Biomed, 108:442-450.

[140] Ponzoni I, Nueda M, Tarazona S, Götz S, Montaner D, Dussaut J, et al. (2014). Pathway network inference from gene expression data. BMC Syst Biol, 8 Suppl 2:S7.

[141] Zafeiris D, Rutella S, Ball GR (2018). An Artificial Neural Network Integrated Pipeline for Biomarker Discovery Using Alzheimer's Disease as a Case Study. Comput Struct Biotechnol J, 16:77-87.

[142] Park C, Kim J, Kim J, Park S (2018). Machine learningbased identification of genetic interactions from 
heterogeneous gene expression profiles. PLoS One, 13:e0201056.

[143] Maj C, Azevedo T, Giansanti V, Borisov O, Dimitri GM, Spasov S, et al. (2019). Integration of Machine Learning Methods to Dissect Genetically Imputed Transcriptomic Profiles in Alzheimer's Disease. Front Genet, 10:726.

[144] Coppedè F, Grossi E, Buscema M, Migliore L (2013). Application of artificial neural networks to investigate one-carbon metabolism in Alzheimer's disease and healthy matched individuals. PLoS One, 8:e74012.

[145] Jamal S, Goyal S, Shanker A, Grover A (2016). Integrating network, sequence and functional features using machine learning approaches towards identification of novel Alzheimer genes. BMC Genomics, 17:807.

[146] Huang X, Liu H, Li X, Guan L, Li J, Tellier LCAM, et al. (2018). Revealing Alzheimer's disease genes spectrum in the whole-genome by machine learning. BMC Neurol, 18:5.

[147] Singhal A, Simmons M, Lu Z (2016). Text Mining Genotype-Phenotype Relationships from Biomedical Literature for Database Curation and Precision Medicine. PLoS Comput Biol, 12:e1005017.

[148] Ye D, Zuo X, Fan C (2018). DNA NanotechnologyEnabled Interfacial Engineering for Biosensor Development. Annu Rev Anal Chem (Palo Alto Calif), 11:171-195.

[149] Koydemir HC, Ozcan A (2018). Wearable and Implantable Sensors for Biomedical Applications. Annu Rev Anal Chem (Palo Alto Calif), 11:127-146.

[150] Haghi M, Thurow K, Stoll R (2017). Wearable Devices in Medical Internet of Things: Scientific Research and
Commercially Available Devices. Healthc Inform Res, 23:4-15.

[151] Zhang MW, Chan S, Wynne O, Jeong S, Hunter S, Wilson A, et al. (2016). Conceptualization of an evidence-based smartphone innovation for caregivers and persons living with dementia. Technol Health Care, 24:769-773.

[152] Kim J, Campbell AS, de Ávila BE, Wang J (2019). Wearable biosensors for healthcare monitoring. Nat Biotechnol, 37:389-406.

[153] Tavassoly I, Goldfarb J, Iyengar R (2018). Systems biology primer: the basic methods and approaches. Essays Biochem, 62:487-500.

[154] Williams AM, Liu 54Y, Regner KR, Jotterand F, Liu P, Liang M (2018). Artificial intelligence, physiological genomics, and precision medicine. Physiol Genomics, 50:237-243.

[155] Brodland GW (2015). How computational models can help unlock biological systems. Semin Cell Dev Biol, 47-48:62-73.

[156] Yang D, Li B (2018). Association, prediction, and engram cells in creative thinking. Cogent Psychol, 5:1493806

[157] Veale Tony, Cardoso FA, editors. Computational Creativity, The Philosophy and Engineering of Autonomously Creative Systems. Basel: Springer International Publishing; 2019.

[158] Carpenter KA, Huang X (2018). Machine Learningbased Virtual Screening and Its Applications to Alzheimer's Drug Discovery: A Review. Curr Pharm Des, 24:3347-3358. 